\title{
Practicing a Record-and-Replay System on USRP
}

\author{
Jian Chen ${ }^{\dagger}$, Shengli Zhang ${ }^{\dagger}$, Hui Wang ${ }^{\dagger}$, Xiufeng Zhang ${ }^{\S}$ \\ †'Shenzhen Key Lab of Advanced Communications and Information Processing, \\ College of Information and Engineering, Shenzhen University, Shenzhen, P.R. China \\ ${ }^{\S}$ Corad Technology Inc., Shenzhen, P.R. China \\ E-mail: chenjian200@126.com, \{zsl,wanghsz $\} @$ szu.edu.cn, roger.zhang @tnmcorad.com
}

\begin{abstract}
Signal recording and replaying is widely used in data analysis, device test, etc. Motivated by this fact, our paper focuses on developing a low-cost record-and-replay prototype based on USRP (Universal Software Radio Peripheral). In particular, we designed and developed a GPS (Global Positioning System) record-and-replay system, which can sample the real GPS signal and then output the analog GPS signal to other devices with cable. In this prototype, we first design the record flow graph and the replay flow graph for USRP, which is verified in the FM band with the FM (Frequency Modulation) broadcasting. After that, we replace the passive antenna with one active antenna suit to amplify the small power GPS signal, lower the quantization bits to reduce the data rate in order to support the wide bandwidth of the GPS signal. Finally, our GPS record-and-replay system is tested and verified by a real GPS receiver. The good performance of our GPS record-and-replay system showed that USRPs can not only used in academia as experiment devices but also used in industry as low-cost wireless auxiliary devices.
\end{abstract}

\section{Categories and Subject Descriptors}

C.2.1 [Network Architecture and Design]: Wireless communication

\section{General Terms}

Experimentation

\section{Keywords}

Record and Replay; USRP; GPS

\section{INTRODUCTION}

Nowadays, many kinds of SDR (Software Defined Radio) hardware platforms were developed: SORA [1], WARP [2], SSRP(Simple Software Radio Peripheral) [3], umtrx [4], Symplex S-Mini [5], Funcube Dongle [6] etc.

Permission to make digital or hard copies of all or part of this work for personal or classroom use is granted without fee provided that copies are not made or distributed for profit or commercial advantage and that copies bear this notice and the full citation on the first page. Copyrights for components of this work owned by others than the author(s) must be honored. Abstracting with credit is permitted. To copy otherwise, or republish, to post on servers or to redistribute to lists, requires prior specific permission and/or a fee. Request permissions from permissions@acm.org.

SRIF'13, August 12, 2013, Hong Kong, China.

Copyright is held by the owner/author(s). Publication rights licensed to ACM.

Copyright 2013 ACM 978-1-4503-2181-5/13/08 ...\$15.00.
USRP (Universal Software Radio Peripheral) is one kind of new SDR hardware platform, a product by Ettus Research [7]. It is very popular for its lower price but acceptable performance, and full compatibility with GNU Radio [8], an open source SDR software. In addition, USRP achieves big success in academic research $[9,10,11,12]$ as an experimental device. But little effert was made to apply USRP in real products: Reference [13] shows a system which uses USRP in a GNSS (Global Navigation Satellite System)[14] Signal Generator, especially in GPS (Global Positioning System, one kind of GNSS). The system can simulate GPS signal, record and replay GPS signal. Reference [15] shows that using USRP to record real GPS signal then using Matlab to decode it.

Recording and replaying real GPS signal is very useful in GPS industry to test the new developed GSP devices. Recently, the ASIC (Application Specific Integrated Circuit) technology of GPS receiver is so mature and successful, that many GPS module or receiver manufactures need corresponding and differential scale instruments to test their products. In fact, many corporations (NAVSYS, NI, Aglient, LabSat, SPRIENT, etc.) have designed and produced the related devices, but the price is high for lower level testing demands.

To satisfy this demand, we decide to implement a lower level device (i.e. a record-and-replay system) for GPS receiver testing. Considering the features of USRP and GNU Radio, we choose them for hardware and software separately. The main but simple idea for a record-and-replay system it to record and replay the raw baseband IQ(In-phase component and Quadrature component) signal, don't need to decode them. We first verify the record and replay function in the band of FM (Frequency Modulation) broadcasting, because FM signal is strong in power level and continuous in time. We then move the system in FM band to GPS band without big change. After that, we add a LNA (Low Noise Amplifier) suit to amplify the GPS signal for the same function testing, due to the fact that the power strength of GPS signal is very weak.

The remaining of this paper is organized by following: Section 2 introduces the system diagram; Section 3 describes system testing; Section 4 makes a conclusion.

\section{SYSTEM DIAGRAM}

This section illustrates the structure of the system, including the details of each block. Figure 1 shows the block diagram of the system. The working process of is as follow: 


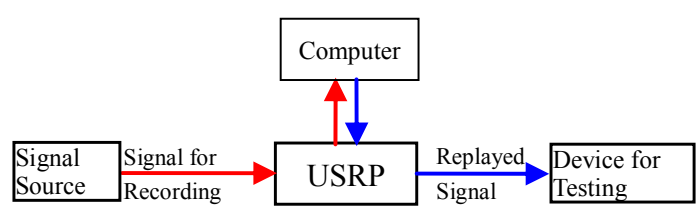

Figure 1: A Record-and-Replay System

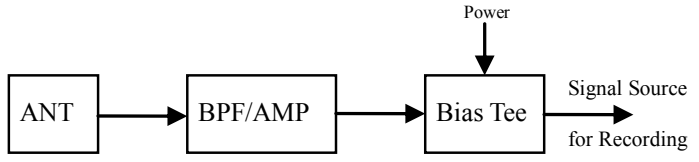

Figure 2: GPS Signal Source

When recording, "Signal Source" provides the signal which we want to record. "USRP" down-converts it to baseband and sampling it, then the data(transferred from the Ethernet cable(for USRP N series)) is stored by the "PC".

When replaying, "PC" send the raw baseband data to the "USRP" through Ethernet cable(for USRP N series), then the "USRP" converts it to analog signal and up-converts to $\mathrm{RF}$ signal. This signal is a reproduced signal which can be used for device testing.

In another words, the red line presents the signal flow for recording and the blue line presents the signal flow for replaying.

From the diagram, we can divide it into three parts: Signal Source; Core Block; Device for Verifying. So, next, we will illustrate them in detail.

\subsection{Signal Source}

In this paper, we only care about FM signal ( $88 \mathrm{MHz} \sim 108$ $\mathrm{MHz})^{1}$ and GPS signal (L1: $\left.1.57542 \mathrm{GHz}\right)^{2}$

For FM signal, The power level is high, and it is continuous in time, so, like general FM receiver, we can use a passive antenna to connect the USRP directly.

For GPS signal, one obvious difference from FM signal is its lower signal strength. It is about $-130 \mathrm{dBm}$ [15]. This means that the signal power is much lower than noise, and the SNR (Signal-to-Noise Ratio) is minus. This low signal is even below the threshold of ADC (Analog-to-Digital Converter) although USRP has on-board PGA(Programmable Gain Amplifier). Besides the noise figure of the PGA is not perfect. By the way, GPS demodulation is using "Direct Sequence Spread Spectrum" to promote the SNR. In our system, it doesn't involve any demodulation process, but for improve the quality of replayed signal, we must reducing adding noise when recording. In one word, we need a "LNA suit" to replace the passive antenna in FM band. Enlighten by Ref.[15], we also use an active antenna to raise the power of the weak signal. However, we use an WBX daughter board which covers the GPS band rather than an RFX900 daughter board in [15]. As a result, the down-converter module is unused.

\footnotetext{
${ }^{1}$ Typical FM band in P.R.China.

${ }^{2}$ Usually, using the civil navigation message in L1 band.
}

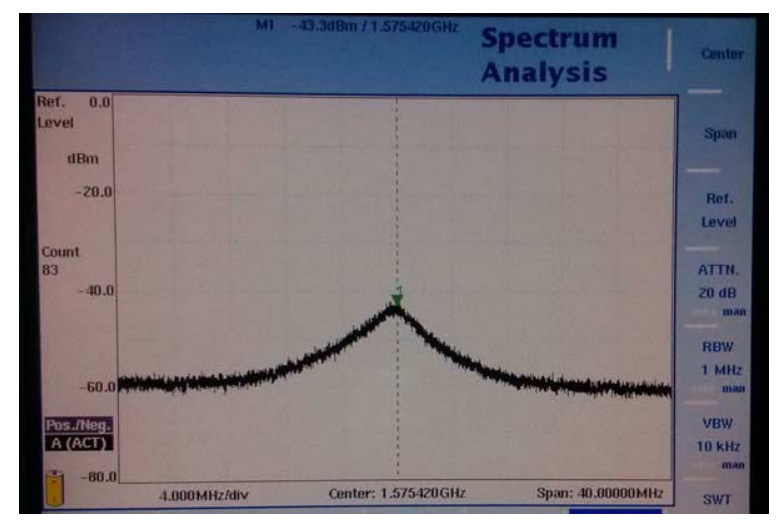

Figure 3: GPS Spectrum for Recording

Figure.2 shows the block diagram about the "LNA suit". The "ANT" can provide $\sim 20 \mathrm{~dB}$ gain, and the "BPF/AMP" can provide $\sim 28 \mathrm{~dB}$ gain. "Bias Tee" is used to supply power for the active antenna("ANT") and "BPF/AMP". In our system, we use AMP28-BT [16] which combines "BPF/AMP" with "Bias Tee". Figure 3 shows the power spectrum of the output of "AMP28-BT". From the figure, We can found that the GPS signal power is much higher with the help of the active antenna suit.

\subsection{Core Module}

The core module includes a USRP N210 with WBX daughter board and a Computer which is installed with GNU Radio. In our system, we choose USRP N210 with WBX daughter board for two reasons: Firstly, for its capacity of high data rate. In theory, USRP N210 supports up to 25 $\mathrm{MHz}$ bandwidth in 16 bits quantization or $50 \mathrm{MHz}$ in 8 bits quantization(restricted by the $1 \mathrm{Gbps}$ rate of the Ethernet interface). This is very useful for wide bind signal record and replay. Secondly, for the cover range of WBX daughter board. The WBX $(50 \mathrm{MHz} \sim 2.2 \mathrm{GHz})$ covers the FM band $(88 \mathrm{MHz} \sim 108 \mathrm{MHz})$ and GPS band (L1: $1.57542 \mathrm{GHz})$. It is convenient for our system.

Specifically, there are some items (sample rate, quantization bits, accuracy of oscillator and data rate) of USRP, which is important for a record-and-replay system.

\subsubsection{Sample Rate}

According to Nyquist Sampling theory: For real sampling, the sample rate should be the twice or larger than the bandwidth of the signal; For complex sampling, the sample rate should be equals to or larger than the bandwidth of the signal. In our system, we use complex sampling. For GPS, from the specific of "ANT" and "AMP28-BT", the bandwidth is $10 \mathrm{MHz}$, which means that the sample rate should be set to $10 \mathrm{MS} / \mathrm{s}$ or higher. For FM case, because there is no $\mathrm{BPF}$ (Band Pass Filter), the higher sample rate we set, the larger bandwidth it covers. But this is restricted by the date rate, will be deeply analysed in the next sub-section "Date Rate".

\subsubsection{Quantization Bits}

In USRP N210, the ADC is 14 bits, in theory, 16-bits data type is enough to represent the data. But for a recordand-replay system, recorded file size is one of the critical parameters. In an addition, for GPS, as described before, 
it uses "Direct Sequence Spread Spectrum" to promote the SNR, and through the quantization bits to do this is limited. Reference [15] analysed the SNR loss to quantization bits. It shows that SNR decrease is negligible when the quantization bits decreased to 4 bits. Through looking up datasheet of GPS ASIC, the quantization bits is always lower than 4 bits, but they have high performance RF front-end. Lower bits can produce smaller file size when recording, but for convenience or easy to use, we choose the 8-bits data type, which is the smallest data type supported by the UHD (Universal Hardware Driver: Driver for USRP).

\subsubsection{Accuracy of Oscillator}

As we known, the frequency correction of GPS receiver is limited. After recording and replaying, it will produce extra and uncertain frequency offset caused by the accuracy of the oscillator. USRP N210 has the on-board TCXO(Temperature Compensated Crystal Oscillator) with $2.5 \mathrm{ppm}^{3}$ accuracy, which is acceptable for the record-and-replay system through testing.

\subsubsection{Date Rate}

For USRP N210, the data rate can not exceed the maximum date rate of Ethernet interface (1Gbps). At the same time, the data rate could not exceed the stable writing speed of hard disk. Omitting the TCP/IP header overhead, the data rate can be calculated by the following formula.

$$
\text { DateRate }=\text { SampleRate } * \text { QuantizationBits } * 2
$$

where "2" means that we use two I/Q components (complex sampling).

In our system, we use $10 \mathrm{MS} / \mathrm{s}$ for sample rate and 8-bits for quantization. This means that "Date Rate" is:

$$
\text { DataRate }=10 \mathrm{MS} / \mathrm{s} * 8 \text { bits } * 2 \approx 20 \mathrm{MB} / \mathrm{s}
$$

This data rate is always below the mainstream hard disk in sale.

When recording, the data rate is persistent. so it is better enlarge the network interface buffer as following ${ }^{4}$, which is hinted by the UHD when running a GNU Radio flow graph. sudo sysctl $-\mathrm{w}$ net.core.rmem $\max =50000000$

sudo sysctl $-\mathrm{w}$ net.core.wmem $\max =1048576$

\subsection{Device for Testing}

To verify the replayed signal, the testing method varies with signal:

For FM, using another USRP to receive the replayed signal is convenient.

For GPS, we choose a GPS receiver (Garmin GPSmap $60 \mathrm{CSx}$ ) to avoid the complicated decoding process.

\section{SYSTEM TESTING}

This section illustrates the system testing issue. Figure 4 and Figure 5 show the GNU Radio flow graphs for recording and replay separately. It is simple, for the fact that it only have a "UHD USRP Source/Sink" module and a "File Sink/Source" module in each flow graph.

Firstly, we verify the record and replay function in the FM band:

\footnotetext{
${ }^{3} \mathrm{ppm}=$ part per million

${ }^{4}$ Linux command for running in terminal
}

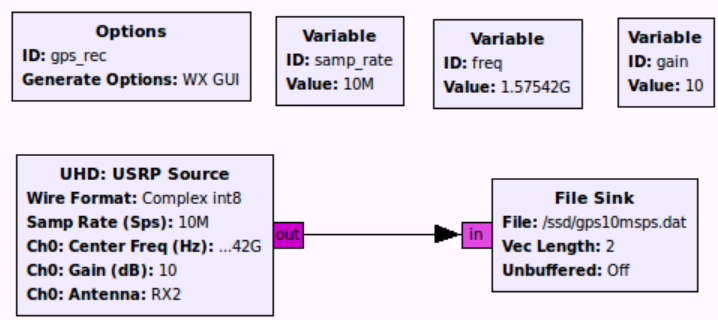

Figure 4: The Flow Graph for Recording

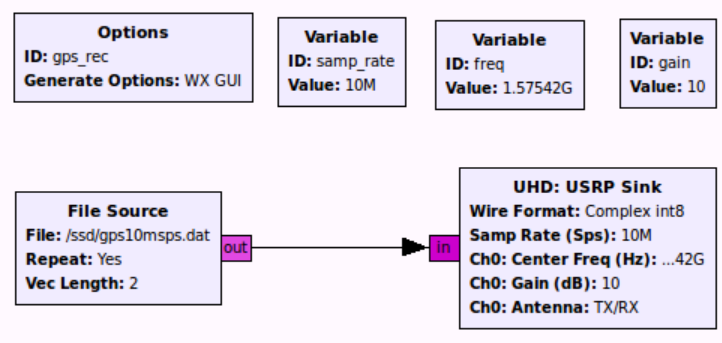

Figure 5: The Flow Graph for Replaying

Recording: Set the center frequency to $97.1 \mathrm{M}$ and set the sample rate to $10 \mathrm{MS} / \mathrm{s}$ in the record flow graph. Connect a passive antenna to the USRP, then run the record flow graph. After a while, stop the flow graph. For testing, several minutes is enough.

Replaying: Set the center frequency to $297.1 \mathrm{M}$ to avoid the real FM signal interference, and set the sample rate to 10 $\mathrm{MS} / \mathrm{s}$ (the same as what is set in the recording flow graph). Using another USRP to receive this replayed FM signal. The result shows that the replayed signal can be decoded correctly, in another words, the decoded audio is clear. This verified the record and replay function.

The FM signal recording and replaying is simple and easy to be verified. so next, extend the testing procedure of recording and replaying function to GPS band:

\subsection{Recording GPS}

It is similar to that in FM band, just replace the active antenna with the "LNA suit"(Figure 2). Connecting this suit to the USRP. In Figure 4, set the center frequency to $1.57542 \mathrm{GHz}$, set the bandwidth to $10 \mathrm{MS} / \mathrm{s}$. Run the flow graph(Figure 4). Normally, GPS receiver need to takes about 30 seconds to acquire satellites, so we record for longer time (about 15 minutes) for testing. Calculate the data amount through Eq.(1), the file size for 15 minutes is about 17 GB. If we check the file size when recording, we found that the file size is became larger as time go on.

\subsection{Replaying GPS}

In Figure 5, select the file name which is just recorded and set the sample rates to $10 \mathrm{MS} / \mathrm{s}$ ( the same as what is set in recording flow graph), set the center frequency to L1 (1.57542 GHz), run the replaying flow graph(Figure.5). Using spectrum analysis to view the replayed signal, shown in Figure 6. Compared with Figure 3, the shapes is a little different, but Figure. 6 is close to what in GPS theory. The main reason for the difference is that we set some gain in 


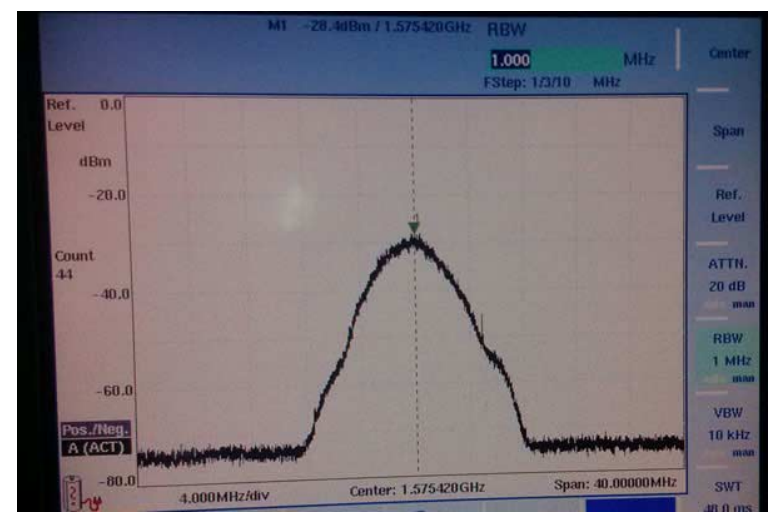

Figure 6: GPS Spectrum after Replaying

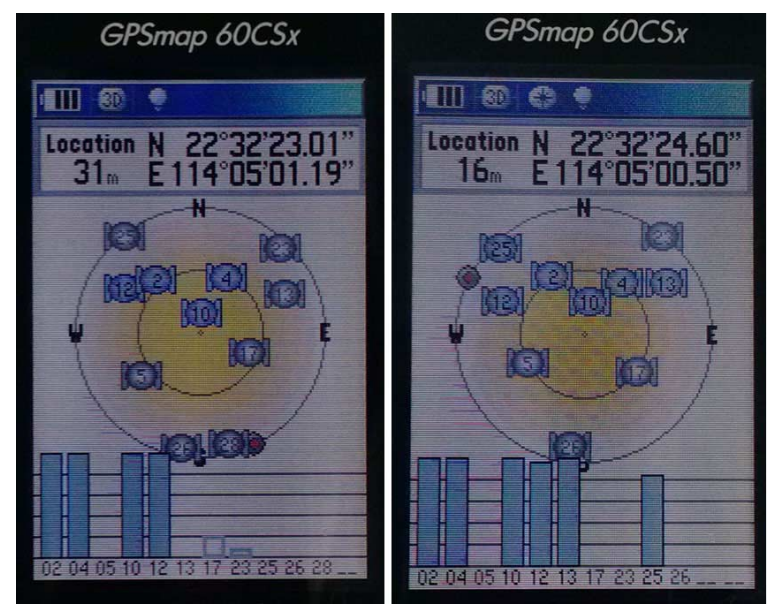

Figure 7: GPS receiver status when replaying

the PGA when recording and replaying. This gain lifts the GPS signal power, and the original shape appeared, for the power is higher than noise.

The next to verify the replayed signal in depth. Take the GPS receiver to do this. For convenience, we connect the USRP and the GPS receiver directly with a cable to avoid the interference from the real GPS signal. Figure 7 captures the GPS receiving status. The figure shows that the GPS receiver can acquire the satellites, and the longitude and latitude are decoded correctly. This proves that the recordand-replay system works well in GPS band.

\section{CONCLUSION}

In this paper, a record-and-replay prototype based on USRP was implemented. Firstly, the record-and-replay system is verified in FM band. After that, we replace the passive antenna with the GPS amplifier suit, then the GPS record and replay function have also been verified. The testing answer shows that the GPS record-and-replay system can be used in industry for its acceptable performance. The system also have space to be promoted: If the quantization bits reduce to 4 bits, it can record double time, but the same file size, and lower date rate for more stable.

\section{ACKNOWLEDGEMENTS}

This paper is partially supported by research grants from Natural Science Foundation of China (Grant No. 60602066, 60773203, 60902016, 61001182 and 61171071), and grants from Foundation of Shenzhen (Grant No. JC201005250034A JCYJ20120613174214967, JCYJ20120613115037732, JC201005250047A，JC201005280404A，JC201005280556A, JC201005250035A and ZDSY20120612094614154).

\section{REFERENCES}

[1] SORA. http: //research.microsoft.com/en-us/projects/sora/.

[2] WARP. http://warp.rice.edu/.

[3] SSRP. http://oscar.dcarr.org/ssrp/.

[4] umtrx. http://code.google.com/p/umtrx/.

[5] Symplex S-Mini. http://gnuradio.org/redmine/ attachments/download/270/05-tao-shd.pdf.

[6] Funcube Dongle. http://www . funcubedongle.com/.

[7] Ettus Research. http://www.ettus.com.

[8] GNU Radio. http://www.gnuradio.org.

[9] S. Katti, S. Gollakota, and D. Katabi. Embracing Wireless Interference: Analog Network Coding. In SIGCOMM '07 Proceedings, pages 397-408. ACM, October 2007.

[10] S. Biddlestone and K. A. Redmill. A GNU Radio Based Testbed Implementation with IEEE 1609 WAVE Functionality. In Vehicular Networking Conference (VNC), 2009 IEEE, pages 1-7, Oct. 2009.

[11] D. Petri, F. Berizzi, M. Martorella, E. D. Mese, and A. Capria. A Software Defined UMTS Passive Radar Demonstrator. In Radar Symposium (IRS), 2010 11th International, pages 1-4, June 2010.

[12] L. Lu, T. Wang, S. C. Liew, and S. Zhang. Implementation of Physical-layer Network Coding. In Communications (ICC), 2012 IEEE International Conference on, pages 4734-4740, June 2012.

[13] A. Brown and B. Johnson. GPS M-Code and P-Code Signal Simulation Using an Open Source Radio Platform, 2011. NAVSYS Corporation.

[14] Global Navigation Satellite System. http: //en.wikipedia.org/wiki/Satellite_navigation.

[15] E. A. Thompson, N. Clem, I. Renninger, and T. Loos. Software-defined GPS receiver on USRP-platform. Journal of Network and Computer Applications, 35:1352-1360, July 2012.

[16] CHBUTC. http://www. chbutc.com. 